\title{
Barriers to multidisciplinary team approach in end-of-life decision-making: Insights from an Indian hospital
}

\author{
Jaydeep Sengupta ${ }^{1}$ and Suhita Chopra Chatterjee ${ }^{2}$ \\ ${ }^{1}$ Sidho-Kanho-Birsha University \\ ${ }^{2}$ Indian Institute of Technology Kharagpur
}

May 4, 2020

\begin{abstract}
A common perception about Multidisciplinary team (MDT) approach is that it improves quality of care to hospitalized patients, particularly those who are dying. However, there is paucity of information on challenges in implementing such approach in end-of-life care (eolc). The present study explores barriers to MDT functioning in the context of 'eolc'. Data collected through interviews of physicians, nurses and other staff, and review of case records of deceased patients of a multispecialty hospital in Kolkata (India) suggest that the MDT approach fails to live up to its rhetoric. One of the major challenges is professional boundary that is intrinsic to MDT. Due to this, specialist physicians fail to make consensus end-of-life decisions by overcoming disciplinary boundaries. Moreover, holistic caregiving is compromised in the physician-dominant medical team, where nurses and other non-clinical caregivers feel marginalized. The hospital environment is also not conducive to multidisciplinary teamwork. Application of a disease-specific treatment protocol in 'eolc' settings aggravates team coordination. 'Eolc' referrals and care transfers becomes very difficult. Dying patients and their families are specially affected by such discordant care. Based on the findings, the study suggests that while team approach is imperative to effective 'eolc', there is a need to shift focus from multidisciplinary to transdisciplinary approach to enhance care integration and patient-centric care to terminally ill patients.
\end{abstract}

\section{Barriers to multidisciplinary team approach in end-of-life decision-making: Insights from an Indian hospital}

\section{Introduction}

In the report,Health Professions Education: A Bridge to Quality', 'cooperation', 'communication', and 'coordination' are identified as essential competencies that all healthcare professionals need to have. It implies the importance of collaborative teamwork in providing comprehensive care. Accordingly, Western countries have implemented this collaborative approach through multidisciplinary teams (MDTs) at all levels of healthcare delivery, drawing membership from professionals across different disciplines and specialties. Besides, physicians, it MDTs also include nurses and other non-clinical healthcare professionals like occupational therapists, spiritual healers, psychosocial counsellors and so on to provide comprehensive and holistic care to patients. There are multiple evidences to support importance of MDT in hospitals. Most promisingly, it decreases length of stay, improves health outcome, enhances inter-personal communication, yields patient satisfaction $^{2,3}$, and reduces workplace burnout of the caregiving staff ${ }^{4}$. A number of palliative care literature also unanimously support multidisciplinary collaboration as a key to holistic care by addressing physical, emotional and spiritual distress of patients at the end-of-life; it also helps formulating comprehensive, personcentric care goals ${ }^{5.6}$. However, few literature also point out drawbacks of multidisciplinary teamwork like physicians' difficulty in fostering long-term relationships with professionals from other disciplines, uneven distribution of work load, unequal recognitions, heterogeneity in orientation of the caregivers and so on ${ }^{7}$. Moreover, in the present time, when disease-specific treatment has emerged as a dominant model of caregiving in biomedicine, maintaining a multidisciplinary approach is difficult. Professional boundaries have 
crept into MDTs, leading to "Invisible boundaries", as Libertii and Gorli ${ }^{8}$ describes the situation where physicians compartmentalize themselves into watertight specialized disciplines leaving no scope for collaboration. Specialist physicians in the capacity of being appointed as the 'primary consultant' usually follow a paternalistic model in planning care goals for the patients, deciding treatment modalities, and unilaterally conveying decisions to other members in the team. To utter dismay, they pay least attention to opinions of other physicians, and also to the preferences of the patients under their supervision. It is as if they own patients $^{9}$. Such an approach adversely affects patient care ${ }^{10-13}$, and is particularly inimical to end-of-life care. Professional boundaries seem to increase the unmet need for palliation, as most specialist physicians feel palliative care not to be their prerogative ${ }^{14}$. Similar results emerge from works of Gardiner et al. ${ }^{15}$ and Powell and Davies ${ }^{16}$.

In India, implementing inter-professional collaboration in caring for dying patients is all the more difficult. Firstly, most hospitals follow a consultative model of palliative care. It means the care is available to terminally ill patients only on referral from the concerned primary; palliative care is not an integral part of treatment protocol. Second, specialist physicians-led MDT have very little representation of non-clinical caregivers like spiritual carers, occupational therapists, community nurses and so on. And finally, unlike West, where MDTs are available at different levels of healthcare delivery system ${ }^{17}$, in India MDT formation is limited to hospital staff, making it highly disadvantageous for the physicians to network with stakeholders beyond institutional boundary ${ }^{18}$. Apart from these few insights, there is lack of information as how 'eolc' is managed in MDTs; what are the challenges and drawbacks. This study presents a number of observations from a multispecialty hospital to address to the information lacunae. It lays special focus on intra/interprofessional boundaries that are potentially disadvantageous to MDTs.

\section{Materials and methods}

The study took a mini-ethnographic approach to collect information on the nature of inter-professional collaboration in a large private hospital and the complexities therein. The sample hospital is a 750-bedded multispecialty facility centre situated in the metro-city of Kolkata (India). It is a nationally reputed institute with branches all over India, and accredited by the Joint Commission International for Patient Safety and Security. Data was collected from June, 2016 to Februray,2017. The researchers studied MDTs that were constituted for terminally ill, geriatric patients during the study period.

Primary data was collected through in-depth interviews and focus group discussions with physicians, nurses, representative members from hospital administration and other staff. A total forty physicians, twenty nurses and ten members from hospital management participated (refer to Table 1: General information about the respondents ). Research participation was restricted within professionals, who were part of the sample MDTs. Besides first-hand data collection, thirty-five case reports were retrospectively reviewed to understand the quality 'eolc' provided to deceased patients. Triage pattern, treatment trajectories, team coordination during care transfers and role of patients' families were given special emphasis. Additionally, overall observation of the hospital procedures also provided valuable insights about MDTs.

The study complied with basic ethical principles laid down by the Indian Council for Medical Research ${ }^{19}$ and the Declaration of Helinsky ${ }^{20}$ regarding obtaining informed consent from the respondents, maintaining confidentiality of data, and presenting cases anonymously. Respondents were adequately informed of the aims, methods, sources of funding, any possible conflicts of interest, institutional affiliation of the researcher, anticipated benefits and potential risks of the study before signing the consent form. Researchers also sought and received ethical clearance from the concerned hospital authorities to gain access inside the premise.

\section{Findings}

Physicians involved in MDTs held diverse opinion on 'eolc'. Many of the opinions were contradictory to one another. The researcher found that conflicts occur when physicians overwhelmingly try to impose their opinion on others in the team. According to the physicians, although most the conflicts were "minor" in nature that got "easily" resolved through appeal to the institutional medical board and arbitration, few were serious that affected interpersonal relationships (refer Table 2: Opinion of the physicians and nurses 


\section{about interpersonal conflicts within MDT ).}

The following sub-sections give a more elaborate account of different barriers of MDTs:

\section{Differences of opinion among the members in MDT}

Differences among senior specialist physicians

Generally, the primary consultant takes the major responsibility to form MDT and coordinate. This includes planning care goals for the concerned patient, making decisions, and sharing treatment-related information with other members. However, it was observed that the primary consultants were more biased in sharing information with physicians from the same discipline than with other participant specialist physicians. Information sharing was rather unilateral with secondary decision-makers having no role other than merely providing consent. In other words, the procedure seemed superficial just to comply with the institutional norm. Physicians, particularly the intensivists felt marginalised in the team, as they were least consulted. This was probably because all the hospitals covered in the study had 'open' type ICUs, where intensivists had no role in decision-making. An intensivist of the hospital said,

Last week a neurologist asked me to attend to a patient that in her opinion was not doing well. I visited the patient, diagnosed, set up a therapeutic plan, and communicated to the neurologist... But, it turned out she [neurologist] completely ignored my opinion! (...) Maybe they (Neurologists) don't consider us (intensivists) important... Another intensivist from the same hospital lamented, "... rather than discussing and agreeing on one solution, everyone tries to impose his/her view, and in the end the most powerful wins." A deeper reflection on the situation suggests that the senior consultants' reluctance to collaborate with the intensivists mainly stemmed from the disciplinary boundaries that existed between them; senior consultants, who were mostly specialists in General medicine, tended to continue treatment for a longer duration than the intensivists. Since the major responsibility of an intensivist is to monitor patients' vital functions, and ensure patient comfort, they claimed to offer holistic care and felt that other specialists had 'narrow-spectrum' of understanding of patients' conditions. Intensivists who were trained in anaesthesia mostly emphasised on patient comfort by managing pain, reducing infection and so on. On the other hand, a neurologist said, [intensivists] are too interventionist! They erratically push and pull tubes! (...) [This morning] a new patient arrived and the intensivist did an ECG mindlessly just because he has to comply with the protocol..." The study found professional boundaries in the hospitals were too stringent to overcome. Another neurologist from the same hospital shared his experience of working in a MDT led by a critical care specialist, I prefer not to strongly impose my opinion on the medical team. It is humiliating to find that my opinion, for most of the time, is not valued by the primary consultants and their subordinates. All I do now is to avoid putting forth my views (...). For instance, in case of brainstem dead patients, I only give opinion to de-escalate aggressive treatment to the team. It is up to them (team) to decide as what to do".

\section{Differences between physicians and nurses}

Lack of team cohesion was not only evident amongst physicians but was also between physicians and nurses. In general, physicians considered nurses to be deficient in knowledge and skills for caring dying patients. A surgeon, who was highly upset with the nursing team said:

"They mess up everything... they don't know how to insert tubes, how to pull them off and how to handle the life-saving equipment... often they end up causing the patient to bleed. I've complained many times to the administration..." Another physician said: "nurses are good in carrying out orders of the doctor, but caring for a dying person means something more than a routine work... they lack the aptitude to identify symptoms of imminent death for a patient. They are unfit to be included in the [medical] decision-making team!!"

Nurses, on the other hand, felt immense difficulty in properly communicating with consultant physicians. They said that the instructions about treatment plans reach nurses through a hierarchic order involving junior physicians, medical officers, and the Head nurse. The nurses were disgusted obeying to a tiring and cumbersome communication hierarchy. They said that the situation becomes 'nightmarish' when terminally 
ill patients suffer from co-morbid conditions. According to an ICU nurse, when a "frail, old" patient suffering from multiple organ failure is admitted to the ICU:

"Different consultants barge into ICU and interfere with treatment procedures. Care goals keep changing with one consultant overriding others' decisions. We are obliged to follow multiple instructions and report to all the concerned physicians... at the end we are confused and the patients distressed. It is totally a chaos for us then..."

Conflictions among nurses of different departments

The nurses of the hospital were also divided on their attitude and perception towards terminally ill geriatric patients. For instance, nurses at the ICU of the Oncology Department said,

"We are very different from others because we have been trained to tackle dying issues. We know when to forego life-saving treatments. In other departments [wards], nurses unquestionably perform whatever the doctors prescribe (...) Death unnerves them...they panic if anything goes against their expectation! I couldn't stand working there... I like working here where I feel doing something independently (referring to supportive care).

A nurse appointed to the High Dependency Unit said,

"No matter how much we claim to have expertise in caring for critically ill patients, physicians consider us subordinates to them. They consider our knowledge and skills to be inadequate

These narratives illustrate the ubiquitous presence of an "invisible boundary" between medical fraternity. Standing on either sides of the boundaries, the professionals vehemently tried defending their standpoints in caring.

\section{Marginalisation of non-clinician caregivers in MDT}

Organisational hierarchy as inimical to team coordination

The hospital promoted a stringent clinical protocol, dominated by specialist physicians, who acted as primary decision-makers during end-of-life, whereas others including registrars, nurses and medical officers, who lacked any sort of specialised clinical knowledge were not allowed participating in decision-making. Ironically, as evident from Table 3 (Distribution of physicians according to their orientation in Palliative \& 'eolc' ) many of these officers had basic orientation in Palliative and End-of-life care, yet they were not empowered to take end-of-life decisions. Social workers, spiritual healers and psychological counsellors appeared nowhere in end-of-life decision-making process.

Geriatrics and Palliative care are extraneous to MDT

MDTs failed to integrate geriatric care even though most of the end-of-life cases pertained to elderly patients - an observation made during the study. This was mainly because many primary consultants, who lead the MDTs, lacked requisite training in geriatric care. Unfortunately, all the three geriatricians of the hospital were appointed as General Physician in the general OPD. According to them, an overwhelming inclination towards disease-specific treatment (or specialised treatment) of the patients cause them to undermine importance of geriatricians for holistic care. Thus geriatricians have least involvement throughout the active treatment trajectory, and also at the end of it when end-stage patients are transferred to palliative care. Moreover, the geriatricians too divided in their opinion about their specific role in the treatment trajectory. While one geriatrician emphasised on their involvement at the initiation of treatment, another laid thrust on continuous engagement and supervision of a patient in the hospital and even post discharge. The third respondent, however, felt a geriatrician's presence is essential at the end of active treatment when complications manifest that other specialists fail to address. Such diversified opinions of the geriatricians make their position fuzzy in the care organisation.

Similar to geriatricians, palliative physicians too had a weak representation in the MDTs. A senior oncologist and a member of a MDT disapproved the need to incorporate palliative care specialist in his team. He strongly 
asserted, "Palliation has no proven efficacy... I usually don't prescribe palliative care". The geriatricians also drew professional boundary between Geriatrics and Palliative care. This was particularly evident from the statement of a geriatrician who said, "palliative care is not my perspective... it means giving up hope". Thus inter-linkages between Palliative care and Geriatrics was grossly overlooked by the physicians leaving a large number of terminally ill geriatric patients in need of palliation to die in despair. It was also observed that a patient-coordinator, who counselled cancer patients throughout the hospital-stay and was immensely invaluable to patients and families for the huge amount of compassion she had for them, was not included in MDT just because she had no formal training either in medicine or nursing.

\section{Implications of conflicts and dissent on end-of-life decisions}

The findings show that hospital culture was barely conducive to consensus decision-making. This had a serious implication on end-of-life decisions. A survey was conducted to inquire how differences in clinical standpoints of the physicians who worked as team members affected end-of-life decision-making. The following sub-sections reveal the adverse consequences:

Different clinical standpoints of specialists affect comprehensive care at the end-of-life

A survey was conducted to understand physicians' opinion about appropriate interventions that could be withdrawn at the end-of-life (refer Table 4: Physicians' opinion on withdrawing treatment/interventions at the terminal stage ). Of the total 40 physicians who were asked to give their opinion, 36 returned filled-up questionnaires.

The table shows physicians differed among themselves in deciding interventions that are to be 'mandatorily withdrawn', 'to be withdrawn after reviewing', 'can be withdrawn if patient wishes' or 'not to be withdrawn at all'. Most of the physicians felt that chemo- and radiotherapy during 'eolc' were redundant at the end-oflife (20 out of 36 physicians were in favour of withdrawing such therapies for all patients at the end-of-life). Similarly, many felt that ICU admissions during end-stage could be avoided (21 out of 36). On the other hand, 50 percent of the physicians (18 out of 36) said that withdrawing Ryle's tube to stop nutrition for the dying patient is unethical. Similarly, 39 percent of the physicians (14 out of 36) did not support the idea of stopping IV hydration. According to these physicians, nutrition and hydration were 'basic' lifesupports. A senior nephrologist said nurses in his hospital were also opposed to the idea of discontinuing food to dying patients. Overall, an important feature that emerges from this survey is that physicians were somewhat reluctant to withdraw interventions that belonged to their own specialty, thus exhibiting a sort of biased attitude in decision-making. Thus, while nephrologists emphasised on dialysis even at the end stage, oncologists opined against discontinuation of chemotherapy and radiotherapy. This information infers how MDTs fail to deliver coordinated care to dying patients.

\section{b. Patients as recipients of discordant care}

Case records, available from the hospital are schematically presented to highlight chaos, conflicts, and tensions that surrounded dying patients in absence of well-coordinated MDTs.

Fig. A: Case of Mrs Soma (70 years; Ca Lung with bone metastasis)

(Figure A)

The case of Mrs. Soma highlights two important issues: firstly, the structured pattern of caregiving in the hospital did not exempt the frail aged dying patient from undergoing aggressive cardio-pulmonary resuscitation (CPR). Moreover, most of the physicians, who were involved in the treatment trajectory of Mrs. Soma, were unfamiliar with her treatment preferences and choices. Such abrupt and short-duration involvement of the physicians with the treatment trajectory possibly made the dying patients feel discomfort. Unfortunately, Soma's preference was only known to the primary physicians, who did not communicate it to the rest of the team members. Worse still, the primary consultant of the patient could not be reached over the phone. This compelled the on-duty physicians to make crucial medical decision of resuscitating the gasping 
patient; it not only dishonoured the family's request to not to resuscitate but violated patient's autonomy also!

\section{Fig. B: Case of Mr Gopal (82 years; COPD and kidney failure)(Figure B)}

In this case, too, the patient was compulsorily subjected to aggressive treatment at the end-of-life. As a result, the patient had to follow strict treatment regime characterised by series of diagnostic tests, ICU admission, and multiple CPR attempts. Unfortunately, the procedure could not save the patient, who eventually died. In fact, Mr. Gopal died without proper end-of-life care. Although he was treated by a team of doctors, none seemed to consider his advanced age and multiple morbidities as criteria to stop aggressive treatment. Moreover, like the previous case he too was transferred from one specialist to another who had little coordination among themselves. Unfortunately, the nephrologist under whose supervision, Mr. Gopal was admitted, was nowhere around during his death. Gopal's family too were not around his deathbed. Such a situation rarely guarantees a continuum of care. It also indicates physicians in their overwhelming enthusiasm to resuscitate patients, ignores symptoms of the impending death.

\section{Fig. C: Case of Mr Kumar (72 years; Metastasis in the right pyriform sinus)(Figure C)}

Mr. Kumar's case also ends with conflict between family members, physicians and the hospital administration on the issue of ICU admission. While the hospital authority and the physicians insisted for ICU admission as per the treatment protocol, the family members strongly opposed the decision. This soon led to chaos and scuffle around the deathbed of the patient. The incident was soon over but took a severe toll on the patients' physical condition who suffered a massive cardiac arrest. None seemed to take into consideration the patient's choice. As a result, Mr. Kumar died alone in the ICU; it was certainly not a 'good death' by any means.

\section{Discussion}

The multispecialty hospital considered for the study largely represents the situation of acute care set-ups across India. An overwhelming interest to form medical teams under leadership of consultant physicians affects interdisciplinary collaboration, a prerequisite for 'eolc'. The above discussion outlines how achieving consensus over end-of-life decisions is a challenge due to reluctance of the physicians to overcome their domain-specific knowledge boundaries. Thus, co-located specialist physicians, does not necessarily guarantee collaborative and comprehensive 'eolc' unless they stop working as independent care providers ${ }^{21}$. However, overcoming inter-professional boundaries is difficult because they are deeply rooted to the biomedical culture that contains divergent approach to 'eolc'. These differences of opinion are detrimental to consensus endof-life decision-making, as evident from table no.4, where physicians fell out in their decisions to withdraw life-sustaining treatment. The finding gets support from global 'eolc' literature that show variations in treatment-limiting decisions owe to physicians' specialisation and level of training ${ }^{22-27}$. Consequently, care goals became ambiguous, unattainable and sometimes violative of rights of patients and families. This is evident from the cases reports of few patients and families, who experienced chaos and humiliation during treatment.

Divergent opinions result in conflict when primary consultants fiercely impose their decisions on others in the team, overriding alternative standpoints. Some researchers also feel that due to an overwhelming urge to uphold the professional superiority, specialist physicians intentionally show little or no respect for other's view. According to Everett C. Hughes, in a medical pluralistic environment, such a phenomenon is common ${ }^{28}$. A similar assertion is also made by Lancaster et al. ${ }^{29}$ according to whom physicians tend to overlook the importance of joint responsibility and are engrossed in asserting their superiority over nurses and other non-clinical professionals. Vazirani, Hays, Shapiro and Cowan ${ }^{30}$ also hold a similar opinion.

Finally, marginalisation of nurses and other non-clinical care providers is a near-universal barrier to physicianled $\mathrm{MDT}^{31}$. The present study is also a testimony to this fact. By over-medicalising 'eolc' protocols, hospitals have made services of nurses, psychological counsellors, spiritual healers, less important. The study also shows how geriatricians and patient coordinators were excluded from MDTs. Unfortunately, elderly 'eolc' patients 
died without receiving age-appropriate care. Considering these barriers, it seems that MDTs are mere assemblage of professionals from different disciplines without necessarily guaranteeing integrated patient care $^{32-38}$. In other words, 'invisible boundaries' within MDTs and disease-specific clinical models make cross-disciplinary communication, relationship-building and comprehensive care unattainable.

From the writing of Choi and $\mathrm{Pak}^{39}$ it seems that the very term MDT is problematic because multidisciplinarity has a narrow approach. It is mainly 'additive' in nature, meaning, that it draws knowledge from all fields but is restricted within boundaries of few disciplines only. Thus, the key to provide concerted service to dying patients in hospitals is to transform MDTs into interdisciplinary teams or, even better, to a transdisciplinary team. They go on explaining that while interdisciplinary approach synthesises knowledge acquired from different disciplines, transdisciplinarity refers to integration of health sciences with social and natural sciences in a humanistic context; it transcends traditional disciplinary boundaries. In the context of palliative care, Piotrowski ${ }^{40}$ briefly outlines evolution of team approach from Traditional Multidisciplinary Practice (a typical physician-led palliative care model where different specialist physicians provide "ad hoc" consultations) to Interdisciplinary Team (also physician-led team but members from different disciplines subsumes to a common organizational framework. However, in reality membership is restricted to physicians and nurses) and finally to Transdisciplinary Team (shared team vision; integrated responsibilities, training, leadership, and decision-making). In fact, the transdisciplinary approach has proven to be effective in improving delivery of palliative and 'eolc'. Daly and Matzel ${ }^{41}$ could successfully recast palliative care in a US-based acute care set-up by forming a transdisciplinary palliative care team composed of physicians, nurses, endof-life counsellors and chaplains. Multiple strategies were adopted to educate the staff on transdisciplinary palliative care and communication. The authors report that adopting transdisciplinary approach resulted in better integration of patient services, early identification of palliative care following hospitalisation, regular assessment of patient's condition, and providing spiritual and social care support to patients and families. Moreover, the caregivers felt lesser workload and burnout.

Transdisciplinary team approach to palliative care in the Indian hospitals can also be adopted. However, given the fact that the physicians and other caregivers lack adequate training in palliative and 'eolc', they first need to undergo a rigorous orientation on the discipline, understanding principles of palliative care. During training, specialist physicians need to be motivated for shifting their attention from disease-specific treatment to person-centric care for end-of-life patients. This is followed by facilitating specialist physicians to network with nurses, non-clinical professionals like psychological and spiritual counsellors, and family members. Sufficient handholding support to the team is necessary to impart skills for leadership, shared decision-making, and conflict resolution. Hopefully, prioritising patient's preference over clinicians' agencies in making medical decisions, including treatment withdrawal, would mitigate difference of opinion between physicians. Finally, to sustain transdisciplinary teams in the hospital it is important that the approach is widely promoted even among healthcare staff working outside the hospital so that the transdisciplinary team approach extends beyond institutional setting and the continuum of care is ensured. Unless these measures are undertaken, adoption of an integrated approach to care for dying patients in hospitals will remain a distant dream.

\section{References}

[1] Knebel E, Greiner AC. editors. Health professions education: A bridge to quality . Washington, D.C: National Academies Press; 2003

[2] Epstein NE. Multidisciplinary in-hospital teams improve patient outcomes: A review. Surgical Neurology International 2014;5(Suppl 7):S295

[3] Saghir NS, Keating NL, Carlson RW, Khoury KE, Fallowfield L. Tumor boards: optimizing the structure and improving efficiency of multidisciplinary management of patients with cancer worldwide.American Society for Clinical Oncology Educational Book2014;34:e461-6

[4] Gorbenko K, Mendelev E, Keefer L. Can multidisciplinary team meetings reduce burnout? Journal of Evaluation in Clinical Practice 2019; June: 1-3. 
[5] Rosen MA, DiazGranados D, Dietz AS, Benishek LE, Thompson D, Pronovost PJ, Weaver SJ. Teamwork in healthcare: Key discoveries enabling safer, high-quality care. American Psychologist2018; 73: 433-450

[6] Vissers, KC, van den Brand MW, Jacobs J, Groot M, Veldhoven C, Verhagen C, ... Engels, Y. Palliative medicine update: a multidisciplinary approach. Pain Practice 2013;13: 576-588

[7] Iliffe S. Myths and realities in multidisciplinary team-working.London Journal of Primary Care 2008; Nov 1;1(2):100-2

[8] Liberati EG, Gorli M, Scaratti G. Invisible walls within multidisciplinary teams, disciplinary boundaries and their effects on integrated care. Social Science EMMedicine 2016; 150: 31-39

[9] McKee M, Healy J. Hospitals in a changing Europe . Philadelphia: Open University Press; 2002

[10] Dixon-Woods M. Why is patient safety so hard? A selective review of ethnographic studies. Journal of Health Services Research and Policy 2010; 15: 11-16

[11] Nugus P, Greenfield D, Travaglia J, Westbrook J, Braithwaite J. How and where clinicians exercise power: interprofessional relations in health care. Social Science $\mathscr{E}$ M Medicine 2010; 71: 898-909

[12] Hewett, DG,Watson BM, Gallois C,Ward M, Leggett BA. Intergroup communication between hospital doctors: implications for quality of patient care. Social Science \& Medicine 2009; 69: 1732-1740

[13] Nembhard, IM, Edmondson AC. Making it safe: the effects of leader inclusiveness and professional status on psychological safety and improvement efforts in health care teams. Journal of Organizational Behavior 2006; 27:941-966

[14] Kennedy R, Abdullah N, Bhadra R, Bonsu NO, Fayezizadeh M, Ickes H. Barriers to effective use of palliative care services in the acute care setting with emphasis on terminal non-cancer diseases. IndianJournal of Palliative Care 2019;25:203-9

[15] Gardiner C, Cobb M, Gott M, Ingleton C. Barriers to providing palliative care for older people in acute hospitals. Age and Ageing 2011: Mar 1;40(2):233-8

[16] Powell AE, Davies HT. The struggle to improve patient care in the face of professional boundaries. Social science $\mathscr{E}$ Medicine 2012; 75: 807-814

[17] Royal College of Nursing and Royal College of General Practice.Report of the Joint Working Party on the Primary Health Care Team1961. London: RGCP

[18] Bowen L. The multidisciplinary team in palliative care: A case reflection. Indian journal of Palliative Care 2014; 20: 142-145

[19] Indian Council of Medical Research. Ethical Guidelines for Biomedical Research on Human . New Delhi: ICMR 2006

[20] World Medical Association. World Medical Association Declaration of Helsinki-Ethical principles for medical research involving human subjects. Nursing Ethics 2002; 9: 105

[21] Imison C, Naylor C, Maybin J. Under one roof: will polyclinics deliver integrated care? London: The King's Fund; 2008

[22] McDonald R, Waring J, Harrison S. Rules, safety and the narrativisation of identity: a hospital operating theatre case study. Sociology of Health \& Illness 2006; 28: 178-202

[23] Cook DJ, Guyatt G H, Jaeschke R, Reeve J, Spanier A, King D, ... Tweeddale M. Determinants in Canadian health care workers of the decision to withdraw life support from the critically ill. Journal of American Medical Association 1995; 273: 703-708

[24] Raffin, TA. Withdrawing life support, how is the decision made? Journal of American Medical Association 1995; 273: 738-739 
[25] Caralis PV, Hammond, JS. Attitudes of medical students, housestaff, and faculty physicians toward euthanasia and termination of life-sustaining treatment. Critical Care Medicine 1992; 20: 683-690

[26] Lega F, DePietro C. Converging patterns in hospital organization, beyond the professional bureaucracy. Health Policy2005; 74: 261-281

[27] Vera A, Kuntz L. Process-based organization design and hospital efficiency. Health Care Management Review 2007; 32:55-65

[28] Zetka Jr, JR. Establishing specialty jurisdictions in medicine: the case of American obstetrics and gynaecology. Sociology of Health $E$ Illness 2011; 33: 837-852

[29] Lancaster, G, Kolakowsky-Hayner, S, Kovacich, J, Greer-Williams. Interdisciplinary communication and collaboration among physicians, nurses, and unlicensed assistive personnel. Journal of Nursing Scholarship $2015 ; 47: 275-284$

[30] Vazirani, S, Hays RD, Shapiro MF, Cowan, M. Effect of a multidisciplinary intervention on communication and collaboration among physicians and nurses. American Journal of Critical Care2005; 14: 71-77

[31] Atwal A, Caldwell K. Do multidisciplinary integrated care pathways improve interprofessional collaboration? Scandinavian Journal of Caring Science 2002; 16: 360-367

[32] Oborn E, Dawson S. Knowledge and practice in multidisciplinary teams, struggle, accommodation and privilege. Human Relations2010; 63:1835-1857

[33] Lewin S, Reeves S. Enacting "team" and "teamwork", using Goffman's theory of impression management to illuminate interprofessional practice on hospital wards. Social Science ES Medicine 2011; 72: 1595-1602

[34] Sanders T, Harrison S. Professional legitimacy claims in the multidisciplinary workplace, the case of heart failure care.Sociology of Health and Illness 2008; 30: 289-308

[35] Waring J, Marshall F, Bishop S. Understanding the occupational and organizational boundaries to safe hospital discharge. Journal of Health Services Research 83 Policy 2015; 20: 35-44

[36] Mackintosh N, Sandall J. Overcoming gendered and professional hierarchies in order to facilitate escalation of care in emergency situations, the role of standardised communication protocols.Social Science and Medicine 2010; 71: 1683-1686

[37] Ferlie E, Fitzgerald L, Wood M, Hawkins C. The nonspread of innovations, the mediating role of professionals. Academy of Management Journal 2005; 48: 117-134

[39] Choi, BC, Pak AW. Multidisciplinarity, interdisciplinarity and transdisciplinarity in health research, services, education and policy: Definitions, objectives, and evidence of effectiveness. Clinical and Investigative Medicine 2006; 29: 351

[40] Piotrowski LF. Transdisciplinary care: The next frontier2017. Available from https://www.nacc.org/vision/julyaugust-2017/transdisciplinary-care-next-frontier/. Accessed $25^{\text {th }}$ March,2020

[41] Daly D, Matzel SC. Building a transdisciplinary approach to palliative care in an acute care setting. OMEGA-Journal of Death and Dying 2013 Aug; 67(1-2):43-51

\section{Acknowledgement}

The authors would like to thank the Ethics committee of Apollo Gleneagles Hospital, Kolkata, India. We are also grateful to all the participating departments, clinicians and nurses of the hospital.

\section{Conflict of interest}

This research has no conflict of interest.

\section{Author contribution}


Dr Jaydeep Sengupta conducted the fieldwork for this study as part of his $\mathrm{PhD}$ programme. The work was supervised by Prof. Suhita Chopra Chatterjee. A part of the main thesis is produced in this coauthored paper, in which Prof. Chopra Chatterjee made a significant contribution, including cross-checking the information, English language correction and editing.

\section{Funding}

Dr J. Sengupta was awarded a full-time studentship to undertake this study by the Indian Institute of Technology Kharagpur.

\section{Hosted file}

Figure_A.docx available at https://authorea.com/users/317500/articles/447578-barriers-to-multidisciplinaryteam-approach-in-end-of-life-decision-making-insights-from-an-indian-hospital

\section{Hosted file}

Figure_B.docx available at https://authorea.com/users/317500/articles/447578-barriers-to-multidisciplinaryteam-approach-in-end-of-life-decision-making-insights-from-an-indian-hospital

\section{Hosted file}

Figure_C.docx available at https://authorea.com/users/317500/articles/447578-barriers-to-multidisciplinaryteam-approach-in-end-of-life-decision-making-insights-from-an-indian-hospital

\section{Hosted file}

Tables 1.docx available at https://authorea.com/users/317500/articles/447578-barriers-to-multidisciplinaryteam-approach-in-end-of-life-decision-making-insights-from-an-indian-hospital

\section{Hosted file}

Tables 2.docx available at https://authorea.com/users/317500/articles/447578-barriers-to-multidisciplinaryteam-approach-in-end-of-life-decision-making-insights-from-an-indian-hospital

\section{Hosted file}

Tables 3.docx available at https://authorea.com/users/317500/articles/447578-barriers-to-multidisciplinaryteam-approach-in-end-of-life-decision-making-insights-from-an-indian-hospital

\section{Hosted file}

Tables 4.docx available at https://authorea.com/users/317500/articles/447578-barriers-to-multidisciplinaryteam-approach-in-end-of-life-decision-making-insights-from-an-indian-hospital 